\title{
Risk-benefit assessment of Bacillus Calmette-Guérin vaccination, anti-phenolic glycolipid I serology, and Mitsuda test response: 10-year follow-up of household contacts of leprosy patients
}

\author{
Sergio Araujo ${ }^{[1],[2],}$ Marina Monteiro Figueiredo Rezende ${ }^{[1]}$, \\ Diogo Carrijo Rodrigues de Sousa ${ }^{[1]}$, Maraísa Resende Rosa ${ }^{[1]}$, \\ Danielle Cristina dos Santos ${ }^{[1]}$, Luiz Ricardo Goulart ${ }^{[1],[2],[3]}$ \\ and Isabela Maria Bernardes Goulart ${ }^{[1],[2]}$
}

[1]. Centro de Referência Nacional em Dermatologia Sanitária e Hanseníase, Hospital de Clínicas, Universidade Federal de Uberlândia, Uberlândia, Minas Gerais, Brasil. [2]. Programa de Pós-Graduação em Ciências da Saúde, Faculdade de Medicina, Universidade Federal de Uberlândia, Uberlândia, Minas Gerais, Brasil. [3]. Instituto de Genética e Bioquímica, Universidade Federal de Uberlândia, Uberlândia, Minas Gerais, Brasil.

\begin{abstract}
Introduction: Despite multidrug therapy, leprosy remains a public health issue. The intradermal Bacillus Calmette-Guérin (BCG) vaccine, Mitsuda test (lepromin skin test), and anti-phenolic glycolipid I (PGL-I) serology are widely used in leprosy studies and have shown great epidemiological value. Methods: This longitudinal study evaluated the relative risks and benefits of these three tools by comparing results observed in household contacts (HHCs) of leprosy patients who developed leprosy with those of HHCs who did not in a population of 2,992 individuals monitored during a 10-year period. Results: Seventy-five (2.5\%) new leprosy cases were diagnosed, including $28(0.9 \%)$ co-prevalent cases. Therefore, for the risk-benefit assessment, $47(1.6 \%)$ HHCs were considered as truly diagnosed during follow-up. The comparison between healthy and affected contacts demonstrated that not only did BCG vaccination increase protection, but boosters also increased to $95 \%$ relative risk (RR) reduction when results for having two or more scars were compared with having no scars [RR, 0.0459; 95\% confidence interval (CI), 0.006-0.338]. Similarly, Mitsuda reactions $>7 \mathrm{~mm}$ in induration presented 7 -fold greater protection against disease development compared to reactions of $0-3 \mathrm{~mm}(\mathrm{RR}, 0.1446 ; 95 \% \mathrm{CI}, 0.0566-0.3696)$. In contrast, anti-PGL-I ELISA seropositivity indicated a 5-fold RR increase for disease outcome (RR, 5.688; 95\% CI, 3.2412-9.9824). The combined effect of no BCG scars, Mitsuda reaction of $<7 \mathrm{~mm}$, and seropositivity to anti-PGL-I increased the risk for leprosy onset 8-fold (RR, 8.109; 95\% CI, 5.1167-12.8511). Conclusions: The adoption of these combined assays may impose measures for leprosy control strategies.
\end{abstract}

Keywords: Epidemiology. Leprosy. Immunology.

\section{INTRODUCTION}

Early diagnosis of and chemotherapy for symptomatic patients are the mainstays of the global control measures to contain leprosy, also known as Hansen's Disease. Despite three decades of multidrug therapy (MDT) treatment and more than 20 years of the World Health Organization (WHO)'s elimination campaign $^{(1)}$, Brazil remains the second most affected country in the world, with 31064 new cases reported in 2014 ${ }^{(2)}$.

Corresponding author: Dr. Sergio Araujo. National Hansen's Disease Programs/ Laboratory Research Branch/School of Veterinary Medicine/Louisiana State University. Skip Bertman Drive, Baton Rouge, 70803 Louisiana, USA.

Phone: 1225 614-8290

e-mail: saraujo@gmx.com

Received 20 July 2015

Accepted 28 September 2015
Currently, there is no specific vaccine for leprosy, which would be the primary method of prevention. Hence, the chemoprophylaxis of contacts is considered to be additional support required to interrupt the chain of transmission, especially in endemic countries where leprosy continues to be a public health concern $^{(3)(4)}$.

Leprosy is a chronic infectious disease that presents a broad immunological spectrum in which the clinical manifestations correlate with the individual type of immune response against the pathogen ${ }^{(5)}$.

There is a balance among genetic and environmental factors and innate and acquired immune responses, by which the resolution of an infection may occur as follows: in susceptible individuals, from a highly bacilliferous disease to the granulomatous containment of infection; or, as expected in individuals not susceptible, spontaneous cure ${ }^{(6)}$.

Intradermic vaccination with Bacillus Calmette-Guérin (BCG), an attenuated strain of Mycobacterium bovis, has been 
widely used across the globe as a preventive strategy against tuberculosis (TB) ${ }^{(7)}$. The Mycobacterium tuberculosis and Mycobacterium leprae bacilli are considered the only human exclusive pathogenic mycobacteria and they share host immune evasion similarities ${ }^{(8)}$. BCG vaccination has been associated with leprosy protection since the $1960 \mathrm{~s}^{(9)}$, and due to common immunopathogenic characteristics between leprosy and TB, the cross-protection against both diseases with modified BCG vaccines continues to be evaluated ${ }^{(10)}$.

Universal BCG immunization is currently applied to all children in Brazil, with boosters given to immediate contacts of new cases of leprosy ${ }^{(11)}$. The cell-mediated immunity (CMI) elicited at the site of inoculation after BCG vaccination is evidenced by a definite scar formation ${ }^{(12)}$.

Household contacts (HHCs) of leprosy patients comprise a recognizable group of individuals who lived in close proximity to a leprosy patient before the patient was diagnosed. There are indications that healthy carriers and individuals with subclinical infection may participate in the dissemination of M. leprae to susceptible persons in endemic communities, and HHCs may be among such carriers. The monitoring of HHCs of leprosy patients also promotes early diagnosis of the disease ${ }^{(6)}$.

The Mitsuda test, or lepromin skin test, is an intradermal injection with a bacilli suspension of heat-killed M. leprae, usually performed during the clinical examination of suspected cases of leprosy. After 21 days, the CMI response elicited against $M$. leprae antigens can be visually observed by the induration at the site of injection. Multibacillary (MB) patients most often do not show any reaction. Paucibacillary (PB) patients usually display positive reactions ${ }^{(13)}$. For the HHCs of leprosy patients, a strong positive response to the Mitsuda test is associated with increased protection against the disease ${ }^{(14)(15)}$.

In contrast, the humoral response with the production of antigen-stimulated antibody against $M$. leprae does not confer resistance to infection. The titration of immunoglobulin $\mathrm{M}$ (IgM) antibodies in serum against phenolic glycolipid I (PGL-I; a species-specific cell wall interface of $M$. leprae) in patients is directly proportional to the bacillary load, with increased levels associated with more severe forms of the disease $\mathrm{e}^{(6)}$ and therefore detected primarily in MB patients.

The serology for antibodies against PGL-I (anti-PGL-I) has also been used to measure the effectiveness of treatment ${ }^{(16)}$. Among HHCs, anti-PGL-I seropositive results indicate a greater risk for disease onset, particularly for the development of MB leprosy ${ }^{(14)(15)}$.

The BCG vaccination, anti-PGL-I serology, and Mitsuda test response have been demonstrated to be excellent epidemiological tools and important assets for monitoring leprosy contacts at higher risk for disease development and post-exposure prophylaxis. This investigation is an extensive comparative analysis of the risk to the relative benefit of these tools in a 10-year follow-up survey with a very large population of HHCs of leprosy patients in an endemic setting.

\section{METHODS}

This study was a longitudinal survey of 2,992 HHCs of leprosy patients who attended the National Reference Center for Sanitary Dermatology and Leprosy (CREDESH) at the Hospital das Clínicas (HC) of the Federal University of Uberlandia (UFU), Minas Gerais, Brazil, during the 10-year period from 2002 to 2013.

A household contact was defined as a person who lives or has lived in the same dwelling as a leprosy patient in the last 5 years prior to the patient's diagnosis.

The CREDESH/HC/UFU is a public health unit with a multi-professional staff that includes physicians, nurses, a physiotherapist, a prosthetist, a pharmacist, a psychologist, social care, a clinical analysis laboratory, and others. The CREDESH/HC/UFU routinely works on leprosy prevention, particularly by monitoring HHCs, actively searching for early cases, and administering treatment to those affected by the disease.

For the purpose of data analysis, the HHCs were stratified according to the clinical classifications of their index cases, as per the Ridley-Jopling classification: TT (tuberculoid), BT (borderline-tuberculoid), BB (mid-borderline), BL (borderline-lepromatous), and LL (lepromatous), and also per operational classifications for $\mathrm{PB}$ or MB.

All HHCs were examined for signs or symptoms of the disease by specialized physicians experienced in leprosy diagnosis and treatment during annual dermato-neurological clinical examinations.

At their first examination, HHCs' vaccination data were assessed according to the presence and number of BCG scars $(0,1$, or 2 scars). HHCs underwent the Mitsuda test (lepromin skin test), with results observed 21-28 days later. Results were stratified according the transverse diameter in millimeters $(\mathrm{mm})$ of the induration (not erythema) at the site of the injection. At the time of the Mitsuda result observation, those individuals without BCG scars or with only one scar received a booster of the $B C G$ vaccine.

The anti-PGL-I enzyme-linked immunosorbent assay (ELISA) was used to detect circulating IgM antibodies in the serum against the M. leprae-native PGL-I molecule and was performed as previously described ${ }^{(16)}$. Results for the antibody titers measured by optical density (OD) at $492 \mathrm{~nm}$ were converted into an ELISA index (EI) $\left(\mathrm{EI}=\mathrm{OD}_{\text {sample }} / \mathrm{OD}_{\text {cut-off }}\right)$, as described previously ${ }^{(4)}$. EI values $>1.1$ were considered positive.

Incomplete data, inconclusive results in any of the tests, and individuals with comorbidities or treatments that could interfere with the results were excluded from the analysis.

Additional inclusion criteria were: a minimum period of 7 years of follow-up, the availability of results for all tests, and medical records with information about the clinical form and operational classification of the index case. Contacts without complete information and those who decided not to participate were excluded from this study. 
Results corresponded to the time point at diagnosis of their index case. For follow-up analysis, contacts were further stratified into two classes: affected (those who developed leprosy) and healthy (those without any sign or symptom of disease).

Among the contacts affected by leprosy, we excluded from the risk-benefit assessment those who presented signs or symptoms at the time of their first examination. That is, the disease was present at the same time point of the diagnosis of the index cases (co-prevalence).

Those who did not present any sign or symptom of the disease at their first examination and developed the disease during follow-up were considered to be truly affected; therefore, their data were applied for the assessment of the association of results with disease outcome.

The research protocol was approved by the institutional review board at the Federal University of Uberlandia (\#099/2003) and was conducted in accordance with the guidelines of the Declaration of Helsinki of 1964 and its later revisions. All participants agreed to take part in this study with written informed consent. Patients and their HHCs that developed the disease received free health care, were examined and classified according to their clinical and operational classifications, and received adequate treatment for their illnesses.

Statistical analyses were performed with BioEstat 5.0 software (Open license). The probability of a disease outcome during the follow-up given the presence of a specific factor was measured by calculating the relative risk (RR) along with the associated $95 \%$ confidence interval $(95 \% \mathrm{CI})$. Significance was set at $\mathrm{p}=0.05$.

\section{RESULTS}

Among the 2,992 HHCs of leprosy patients monitored during the study's 10-year period, $75(2.5 \%)$ new cases were diagnosed. Of these, $57.3 \%(43 / 75)$ were classified at diagnosis as $\mathrm{PB}$ cases, and most $(73.3 \%$; 55/75) presented borderline manifestations. Table 1 shows that forty-seven $(1.6 \% ; 47 / 2992)$ contacts were diagnosed after their second examination during the follow-up and were therefore not considered coprevalent cases. Most HHCs were contacts of MB index cases (84\%; 63/75), particularly contacts of LL cases $(57.3 \%$; 43/75).

The majority of new cases diagnosed among the HHCs $(80 \%$; $60 / 75$ ) occurred during the first year of follow-up, including 28 co-prevalent cases diagnosed at their first examination. Among the 47 contacts that truly developed leprosy during follow-up, $68 \%(32 / 47)$ presented signs or symptoms during the first year of follow-up and 15\% (9/47) in the second and third years.

Among the new cases diagnosed during follow-up, 76.6\% (36/47) had only one BCG vaccination scar at their first examination. These contacts received an extra dose of the BCG vaccine; $80.6 \%$ (29/36) presented signs or symptoms during the first year of follow-up, none in the second year, and, for the next 7 years, one contact was diagnosed per year.
A comparison of the number of BCG scars observed in healthy versus affected contacts demonstrated that the BCG vaccination not only increased protection but also that boosters exponentially increased the protective effect. A stepwise analysis of the RR was performed for the presence of 0,1 , or 2 scars, and the linear regression curve showed a strong correlation $(\mathrm{r}=0.9889)$. The lowest $\mathrm{RR}$ (0.0459; 95\% CI, 0.0062-0.3376) was observed for comparison of the results of individuals with 2 or more scars versus those with none, representing a $95 \% \mathrm{RR}$ reduction for the development of disease in individuals vaccinated twice with BCG (Figure 1A).

Regarding the specific CMI response elicited against heatkilled $M$. leprae observed through the late-hypersensitive response of the Mitsuda test, contacts with induration $>7 \mathrm{~mm}$ had 7-fold greater protection against disease onset than did contacts with indurations of $0-3 \mathrm{~mm}$ (RR, $0.1446 ; 95 \%$ CI, 0.0566-0.3696) (Figure 1B).

The evaluation of the humoral response (Th2) measured by the anti-PGL-I ELISA demonstrated seropositivity of $44.7 \%$ (21/47) among contacts that developed the disease versus $11.8 \%(285 / 2414)$ among those who remained healthy during follow-up, indicating a 5-fold RR increase for disease outcome (RR, 5.688; 95\% CI, 3.2412-9.9824).

Comparing the data obtained from healthy contacts with the data of affected contacts allowed the analysis of the combined association effects of BCG immunization, anti-PGL-I serology, and cell-mediated hypersensitive responses to heat-killed M. leprae with the disease outcome. The risk ratios demonstrated an increased effect towards the onset of disease when a combination of the three assays (BCG scar/Mitsuda test/anti-PGL-I ELISA) was established (Figure 1C). Positive results for both BCG and the Mitsuda test conferred 50\% relative risk reduction (RR, 0.4709; $95 \%$ CI, $0.2500-0.8872$ ), while the combination of the unfavorable results for the three assays (no BCG scar, Mitsuda reaction $<7 \mathrm{~mm}$, and seropositivity to anti-PGL-I) conferred an 8-fold greater risk for the disease onset (RR, 8.1090; 95\% CI, 5.1167-12.8511).

\section{DISCUSSION}

Epidemiological studies in endemic settings are important for assessing the long-term risk and protective factors that may help to prevent the development of a disease. This study reinforces that anti-PGL-I serology, the Mitsuda test, and the BCG vaccination are tools of paramount value in the long-term strategy for further reducing the disease burden due to leprosy.

This study adds evidence and data to observations reported previously by our research group after monitoring the HHCs for five years ${ }^{(14)}$. The maintenance of the new case detection rate at around $2 \%$ among the HHCs indicates the low pathogenicity of M. leprae. Despite exposure, $98 \%$ of HHCs are not affected by the disease, particularly in this endemic region.

The majority of new cases among HHCs occurred during the first year of follow-up, including 28 co-prevalent cases that were diagnosed along with their index cases. This indicates not only that the first examination is important but also that there should be close monitoring during the first year. 
TABLE 1 - Household contacts profile.

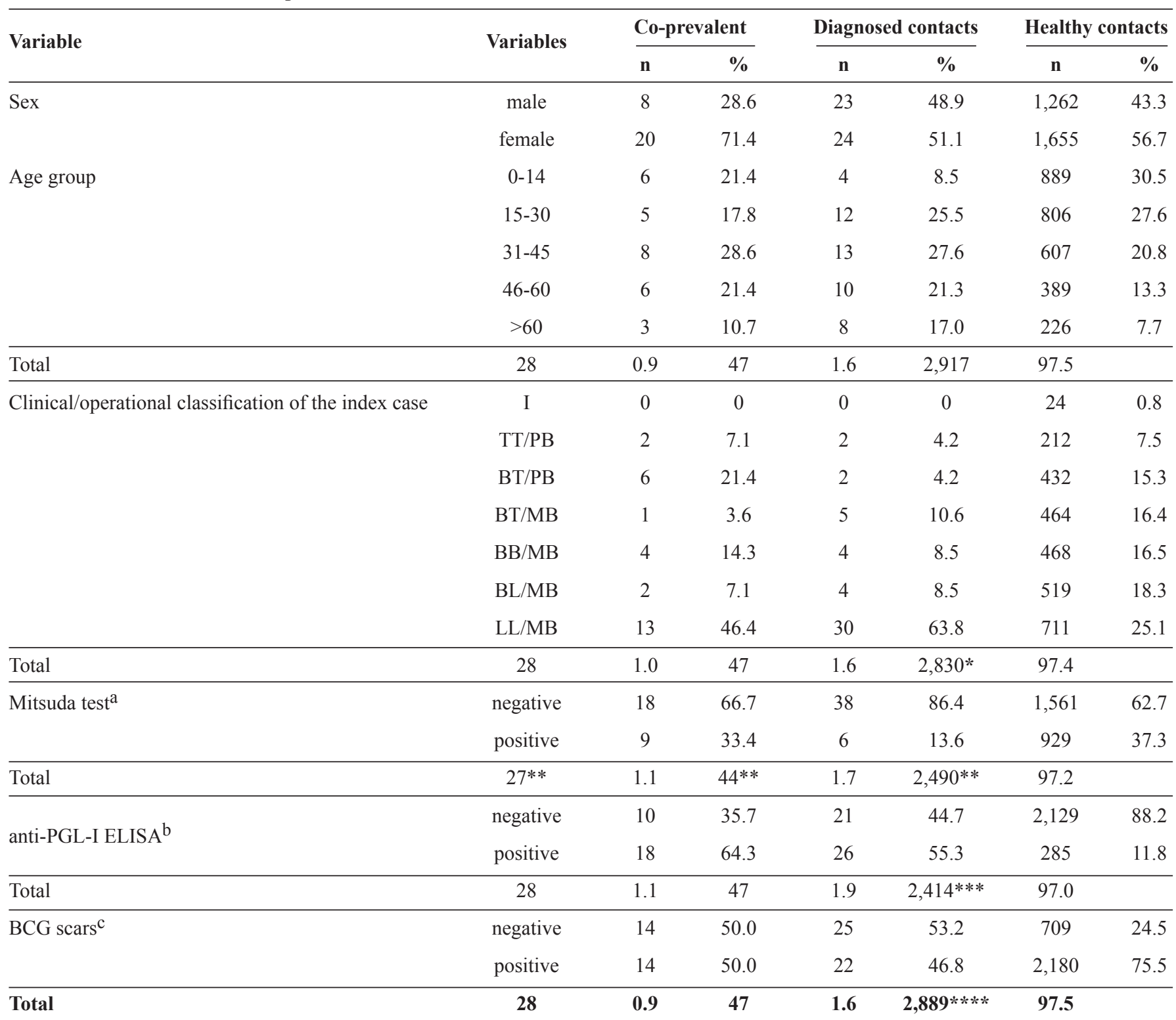

I: Indeterminate leprosy; TT: tuberculoid; PB: paucibacillary; BT: borderline-tuberculoid; MB: multibacillary; BB: mid-borderline; BL: borderline-lepromatous; LL: lepromatous; PGL-I: phenolic glycolipid I; ELISA: enzyme-linked immunosorbent assay; BCG: Bacillus Calmette-Guérin vaccine. CREDESH/UFU: National Reference Center for Sanitary Dermatology and Leprosy/Universidade Federal de Uberlândia. *excluded 87 due to incomplete data; **excluded 431 due to comorbidities or treatments that interfered with results; ***excluded

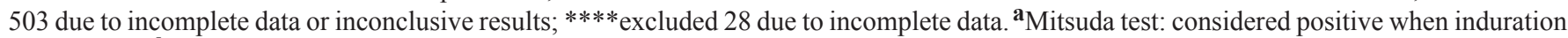

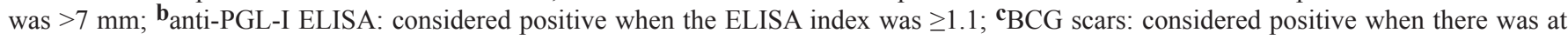
least one scar from the BCG vaccine. Note: Household contacts by sex, age $(\mathrm{N}=2,992)$, clinical and operational classification of the index case ( $\mathrm{N}=2,905)$, Mitsuda test $(\mathrm{N}=2,561)$, anti-PGL-I ELISA $(\mathrm{N}=2,489)$, and presence of BCG scars $(\mathrm{N}=2,964)$. CREDESH/UFU, Brazil, 2013.

On the other hand, we also observed that individuals developed the disease during every year of follow-up, up to the seventh year. Predominantly, the age of affected contacts was between 31 and 45 years. As a chronic disease with a long period of incubation and a common delay reported by new cases between the first symptoms and the diagnosis, the age-specific risk is difficult to assess, especially if occurrence is associated with age at detection rather than with the age at disease onset ${ }^{(17)}$.
As expected, most HHCs that were affected by the disease were contacts of MB index cases. This finding increases the evidence to support closer monitoring of HHCs of MB index cases by the control programs in endemic regions. However, it is noteworthy that the predominant form of illness observed among the HHCs was the PB type of leprosy, with BT clinical manifestations, which are immunologically unstable ${ }^{(13)}$. 
(A)

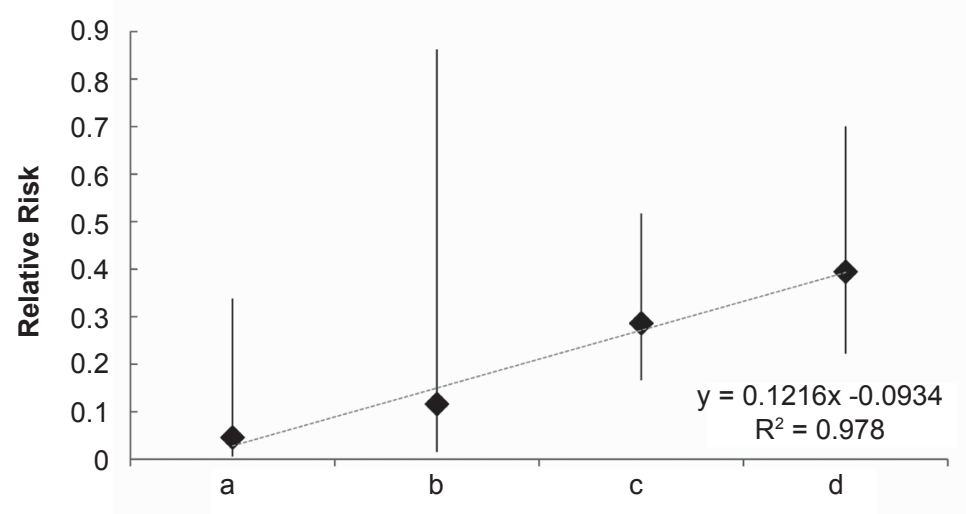

Number of BCG scars

(B)

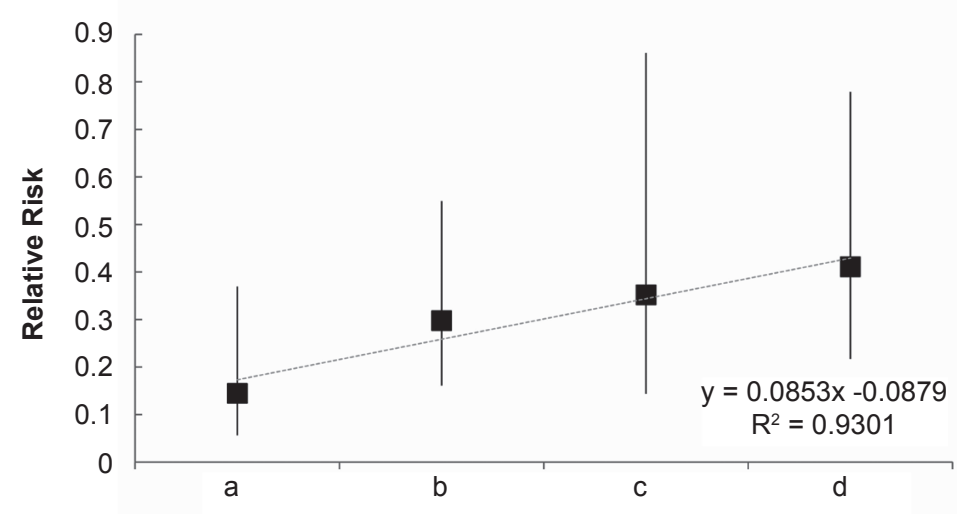

Mitsuda test response

(C)

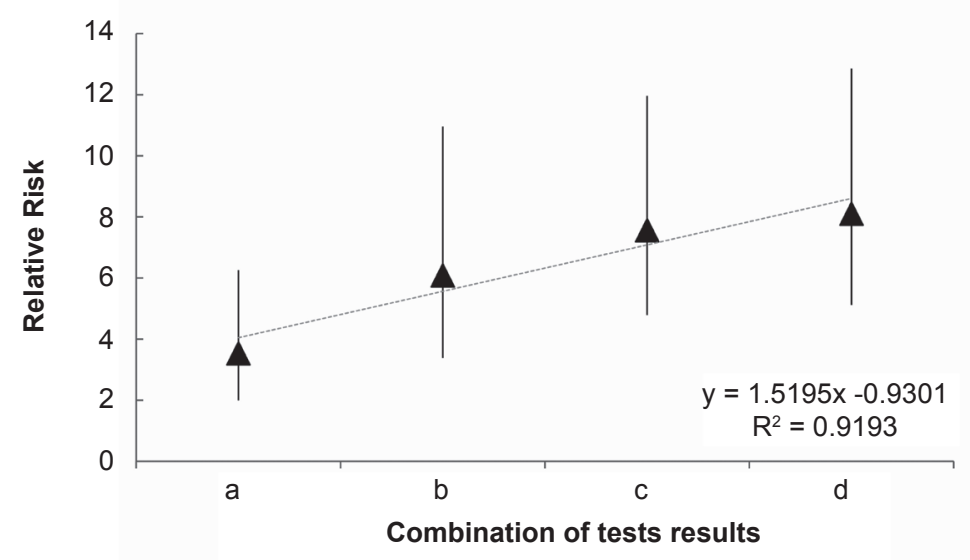

FIGURE 1 - Analysis of the relative risk with 95\% confidence intervals for BCG vaccination, Mitsuda test, and anti-PGL-I serology results observed in healthy household contacts of leprosy patients and in those who developed the disease during follow-up:

A. Number of BCG scars: a. two or more scars versus (vs) no scar (RR, 0.0459; 95\%CI, 0.006-0.338); b. two or more scars vs 1 scar (RR, 0.1162; 95\% CI, 0.0157-0.8622); c. one or more scar vs no scar (RR, 0.2862; 95\% CI, 0.1664-0.5170); d. one scar vs no scar (RR, 0.3947; 95\% CI, 0.2224-0.7004).

B. Mitsuda late-hypersensitivity test: a. induration greater than $(>) 7 \mathrm{~mm}$ vs less than $(<) 4 \mathrm{~mm}(\mathrm{RR}, 0.1446$; 95\% CI, 0.0566-0.3696); b. $\geq 4 \mathrm{~mm}$ vs $<4 \mathrm{~mm}$ (RR, 0.2976; $95 \%$ CI, 0.1612-0.5492); c. $>7 \mathrm{~mm}$ vs $4-7 \mathrm{~mm}$ (RR, $0.3518 ; 95 \%$ CI, $0.1438-0.8606)$; d. $4-7 \mathrm{~mm}$ vs $<4 \mathrm{~mm}$ (RR, 0.4110; 95\% CI, 0.2169-0.7789).

C. Combined results for BCG vaccination, Mitsuda test, and anti-PGL-I serology: a. no BCG scar and Mitsuda $<7 \mathrm{~mm}$ vs all other combination of results (RR, 3.5369; 95\% CI, 1.9946-6.2540); b. Mitsuda $<7 \mathrm{~mm}$ and seropositivity ( + ) to anti-PGL-I vs all other combinations (RR, 6.0895; 95\% CI, 3.3844-10.9567); c. no BCG scar and + to anti-PGL-I vs all other combinations (RR, 7.5684; 95\% CI, 4.7892-11.9603); d. no BCG scar, Mitsuda <7mm, and + to anti-PGL-I vs all other combinations (RR, 8.109; 95\% CI, 5.1167-12.8511).

BCG: Bacillus Calmette-Guérin; anti-PGL-I: anti-phenolic glycolipid I; RR: relative risk; 95\% CI: 95\% confidence intervals. 
The control strategy during the 10 years of this study comprised trained health personnel capable of early leprosy diagnosis and HHC monitoring along with routine BCG immunization and Mitsuda testing. Notwithstanding, those truly susceptible became affected, and regardless of the presumed widespread and continuous exposure of the HHC group, $98 \%$ of them did not develop disease.

The BCG vaccination has been thought to be a prevention strategy against leprosy since the $1960 \mathrm{~s}^{(9)}$, and its great importance as an epidemiological and clinical tool is undeniable. Hence, it is clear that an additional immunization with BCG for HHCs of leprosy patients should be recommended to boost their protection.

That the majority of the new cases were detected among individuals without BCG scars and that they were diagnosed during the first year of follow-up (both of which occurred after inoculation with live BCG and heat-killed M. leprae) raised the hypothesis that the signs and symptoms of the disease were exacerbated after the immune defenses were boosted, either by $\mathrm{BCG}$ or the Mitsuda test, reducing the time for diagnosis and initiation of treatment, and thus the severity of disease.

This survey corroborates with others, indicating that elevated titers of circulating IgM anti-PGL-I are associated with the increased risk for disease onset and that there is a susceptibility linked to immediate contact with MB patients ${ }^{(1)(6)(15)}$. Although they were susceptible, most HHCs that were diagnosed presented less severe clinical manifestations and reduced bacillary burden, suggesting that monitoring contacts effectively improved diagnosis, and also that early cases, even the MB types, exhibit initially subtle signs and symptoms. The initiation of treatment at early stages of the disease prevents nerve damage and incapacitating consequences.

In this extensive analysis over a longer period of time, we reinforced our previous results, which demonstrated that the combination of BCG immunization, the Mitsuda test, and antiPGL-I detection is critical for an active control program and can reduce the severity of the disease, especially regarding HHCs of leprosy patients.

The adoption of these combined assays may impose measures for post-exposure prophylaxis of those at higher risk, i.e., close contacts of newly diagnosed cases. At first, the immediate contacts of every new case should receive immunoprophylaxis, or in some cases, an additional booster of intradermal BCG. Also, serological screening should identify anti-PGL-I seropositive individuals with sub-clinical or asymptomatic leprosy. These individuals should be examined by physicians and followed to confirm if they will or will not develop the disease. To enhance the effectiveness of leprosy control strategies, we suggest that along with these actions, chemoprophylaxis should be administered to all healthy contacts.

\section{ACKNOWLEDGMENTS}

The authors are grateful for the commitment of the staff of the National Reference Center for Sanitary Dermatology and Leprosy (CREDESH) in providing outstanding patient care for the individuals involved in this research.

\section{CONFLICT OF INTEREST}

The authors declare that there is no conflict of interest.

\section{FINANCIAL SUPPORT}

This study was financially supported with grants from: Foundation for Research Support of the State of Minas Gerais [Fundação de Amparo à Pesquisa do Estado de Minas Gerais (FAPEMIG)], Brazilian National Council for Scientific and Technological Development [Conselho Nacional de Desenvolvimento Cientifico e Tecnológico (CNPq)], Brazilian Coordination for Improvement of Higher Education Personnel [Coordenação de Aperfeiçoamento de Pessoal de Nivel Superior (CAPES)], and by the National Fund for Health/Brazilian Ministry of Health.

\section{REFERENCES}

1. World Health Organization (WHO). World Health Assembly (WHA) resolution to eliminate leprosy. Geneva, Switzerland: WHO; 1991.

2. World Health Organization (WHO). Global leprosy update, 2014: need for early case detection. Wkly Epidemiol Rec 2014; 36: 461-476.

3. Goulart IM, Goulart LR. Leprosy: diagnostic and control challenges for a worldwide disease. Arch Dermatol Res 2008; 300:269-290.

4. Schuring RP, Richardus JH, Pahan D, Oskam L. Protective effect of the combination BCG vaccination and rifampicin prophylaxis in leprosy prevention. Vaccine 2009; 27:7125-7128.

5. Goulart LR, Goulart IMB. Leprosy pathogenetic background: a review and lessons from other mycobacterial diseases. Arch Dermatol Res 2009; 301:123-137.

6. Araujo S, Lobato J, Reis EM, Souza DO, Gonçalves MA, Costa $\mathrm{AV}$, et al. Unveiling health carriers and subclinical infections among household contacts of leprosy patients who play potential roles in the disease chain of transmission. Mem Inst Oswaldo Cruz 2012; 107 (supl I):55-59.

7. Abubakar I, Pimpin L, Ariti C, Beynon R, Mangtani P, Sterne JA, et al. Systematic review and meta-analysis of the current evidence on the duration of protection by bacillus Calmette-Guérin vaccination against tuberculosis. Health Technol Assess 2013; 17:1-372.

8. Han XY, Silva FJ. On the age of leprosy. Plos Negl Trop Dis 2014; 8:e2544.

9. Yanagisawa K. On the immunological relationship between tuberculosis and leprosy with special reference to the effect of BCG administration upon the prophylaxis of Leprosy. La Lepro $1960 ; 26: 37-47$

10. Gillis TP, Tullius MV, Horwitz MA. rBCG30-induced immunity and cross-protection against Mycobacterium leprae challenge are enhanced by boosting with the Mycobacterium tuberculosis 30-kilodalton antigen 85B. Infect Immun 2014; 82:3900-3909.

11. Ministério da Saúde. Manual de normas e procedimentos para vacinação. Brasília, Brasil: Ministério da Saúde; 2014.

12. Pereira SM, Dourado I, Barreto ML, Cunha SS, Ichihara MY, Hijjar MA, et al. Sensitivity and specificity of BCG scar reading in Brazil. Int J Tuberc Lung Dis 2001; 5:1067-1070. 
13. Goulart IMB, Penna GO, Cunha G. Immunopathology of leprosy: the complexity of the mechanisms of host imune response to Mycobacterium leprae. Rev Soc Bras Med Trop 2002; 35:365-375.

14. Goulart IMB, Souza DOB, Marques CR, Pimenta VL, Gonçalves MA, Goulart LR. Risk and protective factors for leprosy development determined by epidemiological surveillance of household contacts. Clin Vaccine Immunol 2008; 15:101-105.

15. Reis EM, Araujo S, Lobato J, Neves AF, Costa AV, Gonçalves MA, et al. Mycobacterium leprae DNA in peripheral blood may indicate a bacilli migration route and high-risk for leprosy onset. Clin Microbiol Infect 2014; 20:447-452.

16. Lobato J, Costa MP, Reis EM, Gonçalves MA, Spencer JS, Brennan PJ, et al. Comparison of three immunological tests for leprosy diagnosis and detection of subclinical infection. Lepr Rev 2011; 82:389-401.

17. Noordeen SK. The epidemiology of leprosy. In: Hastings RC, editor. Leprosy. $1^{\text {st }}$ ed. Edinburgh, Scotland: Churchill Livingstone; 1985. p. 15-30. 